\title{
On the efficiency of particle acceleration by rotating magnetospheres in AGN
}

\author{
Z. Osmanov ${ }^{1, \star}$, A. Rogava ${ }^{2,3, \star}$, and G. Bodo ${ }^{4}$ \\ 1 Dipartimento di Fisica Generale, Universitá degli Studi di Torino, via Pietro Giuria 1, Torino 10125, Italy \\ e-mail: osmanov@to.infn.it \\ 2 Centre for Plasma Astrophysics, K.U. Leuven, Celestijnenlaan 200B, 3001 Leuven, Belgium \\ e-mail: Andria.Rogava@wis. kuleuven.be \\ 3 Abdus Salam International Centre for Theoretical Physics, Trieste 34014, Italy \\ e-mail: arogava@ictp.it \\ 4 Osservatorio Astronomico di Torino, Strada dell'Osservatorio 20, 10025 Pino Torinese, Italy \\ e-mail: bodo@to.astro.it
}

Received 13 June 2006 / Accepted 15 January 2007

\section{ABSTRACT}

\begin{abstract}
Aims. To investigate the efficiency of centrifugal acceleration of particles as a possible mechanism for the generation of ultra-high $\gamma$-ray nonthermal emission from $\mathrm{TeV}$ blazars, we study the centrifugal acceleration of electrons by rotating magnetic field lines, for an extended range of inclination angles and determine the maximum Lorentz factors $\gamma_{\max }$ attainable by the electrons via this process. Methods. Two principal limiting mechanisms for the particle acceleration, inverse Compton scattering and breakdown of the beadon-the-wire approximation, are examined.

Results. Particles may be centrifugally accelerated up to $\gamma_{\max } \simeq 10^{8}$ and the main limiting mechanism for the $\gamma_{\max }$ is the inverse Compton scattering.

Conclusions. The energy of centrifugally accelerated particles can be amply sufficient for the generation (via inverse Compton scattering) of the ultra-high energy (up to $20 \mathrm{TeV}$ ) gamma emission in $\mathrm{TeV}$ blazars.
\end{abstract}

Key words. galaxies: active - galaxies: jets - galaxies: nuclei - radiation mechanisms: non-thermal - gamma rays: theory Galaxy: center

\section{Introduction}

One puzzling and interesting problem, related to active galactic nuclei (AGNs) is the origin of the ultra-high $\gamma$-ray emission from blazars. Some blazars, such as Mrk 421, Mrk 501, PKS 2155304, 1ES 2344-514, H1426+428, and 1ES 1959+650, emit TeV $\left(1 \mathrm{TeV} \equiv 10^{12} \mathrm{eV}\right.$ ) photons and form a special class of "TeV blazars" (Dai et al. 2002; Catanese \& Weeks 1999; Horns 2002).

The standard blazar model implies the presence of a supermassive black hole, surrounded by an accretion disk and ejecting twin relativistic jets, one of which is seen almost end-on. The broadband emission spectrum of blazars is made of two components: the low-energy (from radio to optical/UV) part attributed to synchrotron radiation and the high-energy (from $\mathrm{X}$-rays to $\gamma$-rays) component formed by the inverse Compton scattering (ICS) of softer photons (Kino et al. 2002; Kubo et al. 1998). The latter part of the spectrum is usually interpreted on the basis of the synchrotron self-Compton (SSC) model. However, the origin of accelerated and/or pre-accelerated electrons and the mechanism that is responsible for their efficient acceleration is still a matter of uncertainty. Proposed mechanisms such as the Fermi-type acceleration process (Catanese \& Weeks 1999) and re-acceleration of electron-positron pairs as a feedback mechanism (Ghisellini et al. 1993) may account for the observed high

^ On leave from Evgeni Kharadze Georgian National Astrophysical Observatory, Kazbegi ave. 2a, Tbilisi-0160, Georgia. energy emission up to $20 \mathrm{TeV}$. However, the Fermi-type acceleration in relativistic jets is efficient when the seed population of "pre-accelerated" electrons possesses quite high $\left(\gamma_{\max } \geq 10^{2}\right)$ Lorentz factors (Rieger \& Mannheim 2000). The origin of this "pre-acceleration" is still a matter of discussion.

Centrifugally driven outflows (CDOs), centrifugally accelerated particles may acquire quite high energies. This concept (Gold 1968, 1969) has often been discussed (Machabeli \& Rogava 1994; Chedia et al. 1996; Gangadhara 1996; Contopoulos et al. 1999) in the context of pulsar emission theory. Regarding the AGNs it was shown that CDOs from accretion disks occurred if the poloidal magnetic field lines are inclined at an angle $\leq 60^{\circ}$ to the equatorial plane of the disk (Blandford \& Payne 1982).

In the AGN context, the presence of the CDO would imply that despite the intense UV radiation (when via ICS, soft photons are scattered against accelerated electrons and, as a result, electrons lose energy and photons gain energy), electrons may reach quite high, $\gamma_{\max } \sim 10^{5}$, Lorentz factors. If efficient enough, this mechanism could be used not only to justify the pre-acceleration of electrons but it could be considered as an alternative mechanism to generate Blazar high-energy emission (up to $\sim 20 \mathrm{TeV}$ ) (Okumura et al. 2002).

Using the approach suggested by Gangadhara (Gangadhara 1996), where a test charge motion along a rapidly rotating field line was considered in the context of a millisecond pulsar, Gangadhara and Lesch examined the role of the centrifugal force 
on the dynamics of electrons moving along straight magnetic field lines, fixed in the equatorial plane and co-rotating with the spinning AGN (Gangadhara \& Lesch 1997). They have shown that scattering of low-energy photons against accelerated electrons may lead to the generation of the nonthermal X-ray and $\gamma$-ray emission.

This problem was re-examined by Rieger and Mannheim (Rieger \& Mannheim 2000) who tried to specify whether the rotational energy gain of charged particles, moving along straight magnetic field lines, is limited not only by ICS but also by the breakdown of the-bead-on-the-wire approximation (BBW). The latter would happen in the vicinity of the light cylinder when a Coriolis force acting on the particle and trying to "tear it off" the field line would exceed the Lorentz force binding the particle to the field line. According to their consideration, the maximum value of the Lorentz factor, being the subject of both limitations, is $\gamma_{\max } \sim 1000$, which is not enough to produce the ultra-high energy photons emitted by TeV blazars.

In real, three-dimensional astrophysical situations (for example jets) the magnetic field lines are not localized in the equatorial plane but are inclined with respect to it. In this paper we re-examine the same problem considering the wide range of possible inclinations. We show that, for a wide range of AGN, the mechanism responsible for limiting the attainable maximum Lorentz factors is ICS, which under certain conditions can allow particles to reach quite high Lorentz factors $\gamma_{\max } \geq 10^{5}$. The BBW becomes important for the low luminosity $\left(<10^{41} \mathrm{erg} / \mathrm{s}\right)$ AGN, when $\gamma_{\max } \sim 10^{8}$. For higher luminosities $\left(>10^{41} \mathrm{erg} / \mathrm{s}\right)$ it can be dominant only for relatively small inclinations of the magnetic field lines with respect to the rotation axis $\left(\leq 10^{\circ}\right)$. Therefore, contrary to the conclusions of Rieger and Mannheim (Rieger \& Mannheim 2000), we argue that CDOs could be efficient enough to account for the TeV blazar emission.

The paper is arranged in the following way: in Sect. 2 we derive basic equations. In Sect. 3 we consider a case when magnetic field lines are located in the equatorial plane and show that the presence of the ICS allows a wide range of AGN to generate ultra-high photon emission. In Sect. 4 inclined magnetic field lines are considered. We show that in this case ICS still remains the dominant limiting mechanism for most of the AGN. In the final section we summarize the obtained results.

\section{Main consideration}

We consider a typical AGN with a central black hole mass $M_{\mathrm{BH}}=10^{8} M_{\odot}$ and an angular rate of rotation $\omega \sim 3 \times 10^{-5} \mathrm{~s}^{-1}$ (that is only $1 \%$ of the maximum possible rotation rate for black holes with this mass), which makes the light cylinder radius located at $r_{\mathrm{L}} \approx 10^{15} \mathrm{~cm}$. The values of the angular velocity and corresponding light cylinder radius are typical for AGN winds (Belvedere et al. 1989; Rieger \& Mannheim 2000). This spinning rate corresponds to the Keplerian radius $\sim 8 \times R_{\mathrm{g}}$ (where $R_{\mathrm{g}} \equiv 2 G M_{\mathrm{BH}} / c^{2}$ is the gravitational radius) which is about where softer photons are expected to originate (Peterson 1997); these photons are instrumental for the Inverse Compton mechanism.

Rieger \& Mannheim (2000) consider two limiting mechanisms for the maximum Lorentz factor: ICS, when soft photons are scattered against electrons gaining energy and suspending further electron acceleration and $\mathrm{BBW}$ when due to the violation of the balance between the Coriolis and Lorentz forces, acting on the electron, it leaves the magnetic field line.

When the electron moves along the rotating magnetic field line it experiences the centrifugal force and as a result accelerates. The corresponding time scale describing the acceleration process can be defined as (Rieger \& Mannheim 2000):

$t_{\mathrm{acc}} \equiv \frac{\gamma}{\mathrm{d} \gamma / \mathrm{d} t}$

The acceleration lasts until the electron encounters a photon, which may limit the maximum Lorentz factor of the particle. This process may be characterized by the corresponding cooling time scale (e.g. Rybicki \& Lightman 1979; Rieger \& Mannheim 2000):

$t_{\text {cool }}=3 \times 10^{7} \frac{\gamma}{\left(\gamma^{2}-1\right) U_{\text {rad }}}[\mathrm{s}]$,

where $U_{\text {rad }}=\tau l_{\mathrm{e}} \times L_{\mathrm{Edd}} / 4 \pi c R^{2}$ is the energy density of the radiation, $\gamma$-the Lorentz factor of the electron, $\tau \leq 1, L_{\mathrm{Edd}}$ is the Eddington luminosity (for the mentioned mass of AGN $L_{\text {Edd }} \approx$ $\left.10^{46} \mathrm{erg} / \mathrm{s}\right), l_{\mathrm{e}} \equiv L / L_{\text {Edd }}\left(L\right.$ is a disk luminosity), $10^{-7} \leq l_{\mathrm{e}} \leq 1$. In Rieger \& Mannheim (2000) the range $10^{-4} \leq l_{\mathrm{e}} \leq 1$ was used but in this paper we consider the whole range of AGN luminosities (down to AGN with the lowest luminosities $10^{39} \mathrm{erg} / \mathrm{s}$ $\left(l_{\mathrm{e}} \approx 10^{-7}\right)$ (Jimenez-Bailon et al. 2003).

Let us start by the general case when straight magnetic field lines are inclined by the angle $\theta$ to the rotation axis and supposing that magnetic field lines remain straight up to the light cylinder. Let us introduce a metric in the co-moving frame of reference

$\mathrm{d} s^{2}=-c^{2}\left(1-\frac{\omega^{2} R^{2} \sin ^{2} \theta}{c^{2}}\right) \mathrm{d} t^{2}+\mathrm{d} R^{2}$,

derived from the Minkowskian metric $\left(\mathrm{d} s^{2}=\eta_{\alpha \beta} \mathrm{d} x^{\alpha} \mathrm{d} x^{\beta}\right.$, with $\eta_{\alpha \beta} \equiv \operatorname{diag}\{-1,+1,+1,+1\}$ and $\left.x^{\alpha} \equiv(c t ; x, y, z)\right)$ after the following variable transformation: $x=R \sin \theta \cos \omega t, y=$ $R \sin \theta \sin \omega t$ and $z=R \cos \theta$.

Defining $\Omega=\omega \sin \theta(3 \mathrm{a})$ reduces to:

$\mathrm{d} s^{2}=-c^{2}\left(1-\frac{\Omega^{2} R^{2}}{c^{2}}\right) \mathrm{d} t^{2}+\mathrm{d} R^{2}$,

which formally coincides with the metric in the co-moving frame of reference in the case of the inclination angle $\theta=90^{\circ}$ (Machabeli \& Rogava 1994). This means that all kinematic expressions valid for the equatorial plane are also valid for the inclined trajectories of electrons if instead of $\omega$ we use $\Omega$. For the equation of motion, from ( $3 b)$, we get:

$\frac{\mathrm{d}}{\mathrm{d} \tau} \frac{\partial L}{\partial \dot{\bar{x}}^{\alpha}}=\frac{\partial L}{\partial \bar{x}^{\alpha}}$

$L=-\frac{1}{2} m c \bar{g}_{\alpha \beta} \frac{\mathrm{d} \bar{x}^{\alpha}}{\mathrm{d} \lambda} \frac{\mathrm{d} \bar{x}^{\beta}}{\mathrm{d} \lambda}$

$\bar{g}_{\alpha \beta} \equiv \operatorname{diag}\left\{-\left(1-\frac{\Omega^{2} R^{2}}{c^{2}}\right), 1\right\}$

$\bar{x}^{\alpha} \equiv(c t ; R), \dot{\bar{x}}^{\alpha} \equiv \frac{\mathrm{d} \bar{x}^{\alpha}}{\mathrm{d} \lambda}$.

According to this approach all physical quantities are functions of a parameter $\lambda$. Then from Eqs. (4a) for $\alpha=0$ using the four velocity identity $\bar{g}_{\alpha \beta}\left(\mathrm{d} \bar{x}^{\alpha} / \mathrm{d} \lambda\right)\left(\mathrm{d} \bar{x}^{\beta} / \mathrm{d} \lambda\right)=-1$ one can express the Lorentz factor of electrons as a function of the radial distance (Machabeli \& Rogava 1994):

$\gamma=\frac{1}{\sqrt{\tilde{m}}\left(1-\frac{\Omega^{2} R^{2}}{c^{2}}\right)}$. 
Combining Eqs. (1) and (5), for the acceleration time scale we have (Rieger \& Mannheim 2000):

$t_{\mathrm{acc}}=\frac{c \sqrt{1-\frac{\Omega^{2} R^{2}}{c^{2}}}}{2 \Omega^{2} R \sqrt{1-\widetilde{m}\left(1-\frac{\Omega^{2} R^{2}}{c^{2}}\right)}}$,

where $\widetilde{m}=\left(1-\Omega^{2} R_{0}^{2} / c^{2}-v_{0}^{2} / c^{2}\right) /\left(1-\Omega^{2} R_{0}^{2} / c^{2}\right)^{2} . R_{0}$ and $v_{0}$ are initial position and initial radial velocity of the particle, respectively.

To evaluate the efficiency of each of the limiting mechanisms one needs the expression for the maximum Lorentz factor attainable by an electron subject to ICS. Initially the electrons accelerate and this process lasts until the energy gain is balanced by the energy losses due to ICS. This happens when $t_{\text {acc }} \simeq t_{\text {cool }}$. From Eqs. (2), (5), and (6) it follows that near the light cylinder, when $\Omega R / c \rightarrow 1$ (and $\gamma \rightarrow \infty$ ) both time scales tend to zero, which means that ICS starts working efficiently in the vicinity of the light cylinder, hampering the subsequent acceleration of the electron. Using the corresponding condition for time scales:

$t_{\mathrm{cool}} \approx t_{\mathrm{acc}}$

and combining Eqs. (2), (6), and (7) one may easily derive an approximate expression for the maximum Lorentz factor:

$\gamma_{\max }^{\mathrm{ICS}} \approx 10^{14} \sqrt{\widetilde{m}}\left[\frac{6 \Omega}{U_{\mathrm{rad}}\left(R_{\mathrm{L}}\right)}\right]^{2}$,

where $R_{\mathrm{L}} \approx r_{\mathrm{L}} / \sin \theta$.

In order to derive the analogous estimation of the maximum Lorentz factor limited by the BBW mechanisms we can use the method developed by Gangadhara (Gangadhara 1996). For this purpose we use the force responsible for the BBW. The particle momentum in the laboratory frame of reference is:

$\boldsymbol{P}=m v_{r} \boldsymbol{e}_{r}+m v_{z} \boldsymbol{e}_{z}+m \omega \times \boldsymbol{R}$,

where $v_{r}=\sin \theta(\mathrm{d} R / \mathrm{d} t)$ and $v_{z}=\mathrm{d} z / \mathrm{d} t$.

Differentiating this equation and taking into account $\mathrm{d} \boldsymbol{e}_{i} / \mathrm{d} t=$ $\omega \times \boldsymbol{e}_{i}, i=r, z$ :

$\frac{\mathrm{d} \boldsymbol{P}}{\mathrm{d} t}=\left(\frac{\mathrm{d} \boldsymbol{P}}{\mathrm{d} t}\right)_{n}-m \omega^{2} r \boldsymbol{e}_{r}+\omega\left(m v_{r}+\frac{\mathrm{d}(m r)}{\mathrm{d} t}\right) \boldsymbol{e}_{\varphi}$,

where $\left(\frac{\mathrm{d} \boldsymbol{P}}{\mathrm{d} t}\right)_{n}$ denotes the time derivative of the momentum defined in the non-inertial frame of reference.

For the inertial forces (taking into consideration the relationship: $r=R \sin \theta$ ) we have:

$\boldsymbol{F}_{\text {in }}=m \omega^{2} R \sin \theta \boldsymbol{e}_{r}-\Omega\left(m v+\frac{\mathrm{d}(m R)}{\mathrm{d} t}\right) \boldsymbol{e}_{\varphi}$.

Generally the force responsible for BBW is the projection of $\boldsymbol{F}_{\text {in }}$ on the direction perpendicular to the magnetic field line:

$F_{\perp}=\left[F_{\mathrm{inr}}^{2} \cos ^{2} \theta+F_{\mathrm{in} \varphi}^{2}\right]^{1 / 2}$,

where

$F_{\text {inr }}=m_{0} \gamma \omega^{2} r \sin \theta$

$F_{\text {in } \varphi}=-m_{0} \Omega\left(\gamma v+\frac{\mathrm{d}(\gamma R)}{\mathrm{d} t}\right)$.
Note that (12a), in the limit $\theta=90^{\circ}$, reduces to the expression (Rieger \& Mannheim 2000):

$F_{\perp}=m_{0} \omega\left(2 \gamma \frac{\mathrm{d} R}{\mathrm{~d} t}+R \frac{\mathrm{d} \gamma}{\mathrm{d} t}\right)$

coinciding with the Coriolis force acting on the particle.

While the electron moves along the magnetic field line, apart from the inertial forces, it also experiences the Lorentz force:

$\boldsymbol{F}_{\mathrm{L}}=\frac{q}{c} \boldsymbol{v}_{\text {rel }} \times B$

where $v_{\text {rel }}$ is the velocity of the electron relative to the magnetic field line and $m_{0}$ and $q$ are the electron's rest mass and charge, respectively. By virtue of the Lorentz force, the electron gyrates around the magnetic field line and, during the course of motion, the Lorentz force changes from parallel to antiparallel to $\boldsymbol{F}_{\perp}$. If the Lorentz force is greater than $F_{\perp}$ the particle moves along the magnetic field line. The situation changes when $F_{\perp}$ exceeds $F_{\mathrm{L}}$, in this case $\boldsymbol{F}_{\perp}$ forces the particle to come off the magnetic field line. Therefore, the assumption that the particle sticks to the field line is no longer valid and the BBW becomes important as a limiting mechanism for the centrifugal acceleration.

From the condition $F_{\mathrm{L}} \approx F_{\perp}$ (Rieger \& Mannheim 2000) one can derive an estimate of the maximum Lorentz factor limited by the BBW:

$\gamma_{\max }^{\mathrm{FB}} \approx A_{1}+\left[A_{2}+\left(A_{2}^{2}-A_{1}^{6}\right)^{1 / 2}\right]^{1 / 3}+\left[A_{2}-\left(A_{2}^{2}-A_{1}^{6}\right)^{1 / 2}\right]^{1 / 3}$,

where

$A_{1}=-\frac{\operatorname{ctg}^{2} \theta}{12 \widetilde{m}^{1 / 2}}$

$A_{2}=\frac{q^{2} l_{\mathrm{e}} \times L_{\mathrm{Edd}}}{4 m_{0}^{2} \widetilde{m}^{1 / 2} c^{5}}+A_{1}^{3}$

which in the limit $\theta=90^{\circ}$ reduces to the form given in (Rieger \& Mannheim 2000):

$\gamma_{\max }^{\mathrm{BBW}} \approx \frac{1}{\widetilde{m}^{1 / 6}}\left(\frac{B\left(r_{\mathrm{L}}\right) q}{2 m \omega c}\right)^{2 / 3}$,

where $B(r)$ is the equipartition magnetic field strength (which means that magnetic field and radiation energy densities are equal) at the radius $r$ and is given by the formula (Rieger \& Mannheim 2000):

$B^{2}(r)=\frac{2 l_{\mathrm{e}} \times L_{\mathrm{Edd}}}{r^{2} c}$.

\section{The case of magnetic field lines fixed in the equatorial plane}

We start by considering the case when $\theta=90^{\circ}$. From comparison of graphs of $\gamma_{\max }^{\mathrm{BBW}}(L)$ and $\gamma_{\max }^{\mathrm{ICS}}(L)$ (see Fig. 1) one can see that for AGN with luminosities $L>8 \times 10^{40} \mathrm{erg} / \mathrm{s}$ the maximum Lorentz factor attainable by electrons via ICS (solid line) is less than the corresponding Lorentz factor attainable via BBW (dashed line), which means that the latter is unimportant and it becomes significant only for objects with $L<8 \times 10^{40} \mathrm{erg} / \mathrm{s}$. From Fig. 1 it is also clear that, for $L>8 \times 10^{40} \mathrm{erg} / \mathrm{s}$, increasing the luminosity power, the maximum attainable Lorentz factors decrease. From Eq. (8) we also can see that the maximum Lorentz factors scale as $\gamma_{\max }^{\mathrm{ICS}} \sim 1 / U_{\text {rad }}^{2}$, as a result of the dependence of $t_{\text {cool }}$ on $U_{\text {rad }}$. The conclusion made by Rieger and 


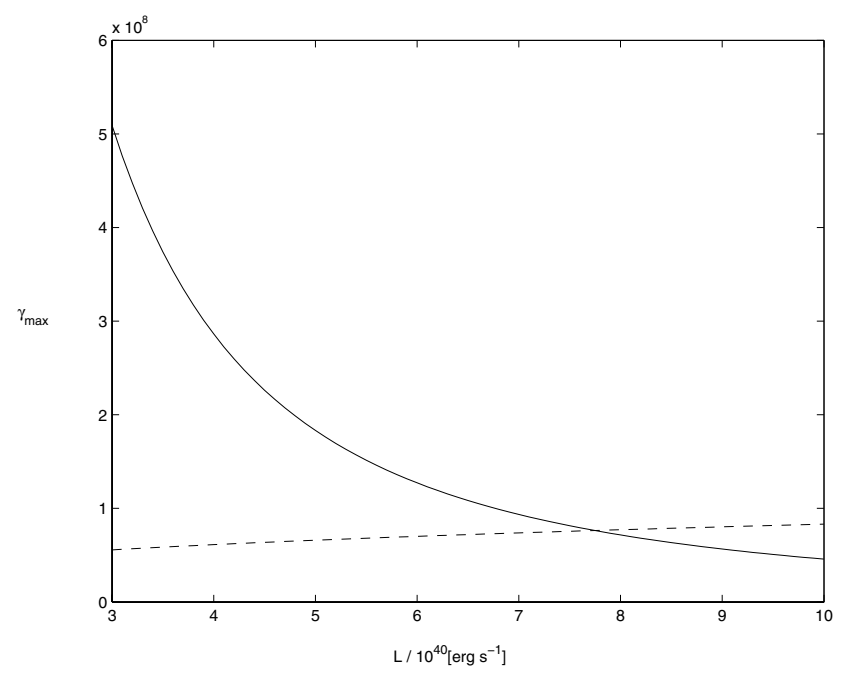

Fig. 1. Graphs of dependence of maximum Lorentz factors on $L . \gamma_{\max }^{\mathrm{ICS}}$ and $\gamma_{\max }^{\mathrm{BBW}}$ are presented by the solid line and the dashed line respectively. The set of parameters is: $\tau=1, \omega r_{0} / c=0.4, v_{0} / c=0.6$ and $\omega=3 \times 10^{-5} \mathrm{~s}^{-1}$.

Mannheim (Rieger \& Mannheim 2000) is quite different. These authors estimated the upper limit of the maximum Lorentz factor attainable under the BBW for $l_{\mathrm{e}}<10^{-3}$ (when according to Rieger \& Mannheim (2000) ICS is unimportant) as $\gamma_{\max } \sim$ 1000 and even for the highest possible magnetic field strength $-B\left(r_{\mathrm{L}}\right)=100 G-$ as $\gamma_{\max } \sim 2500$. The correct value of the corresponding Lorentz factor is $\gamma_{\max } \sim 4 \times 10^{8}$. This circumstance changes the result of Rieger and Mannheim, because this value of $\gamma_{\max }$ is larger by many orders of magnitude, than the corresponding limit of $\gamma_{\max }$ given by ICS (equal, in this case, to $\left.\sim 3.6 \times 10^{3}\right)$. This means that the latter is dominant and the lower limit of $l_{\mathrm{e}}$ at which the ICS still works must be shifted from $10^{-3}$ to $8 \times 10^{-6}$ (see Fig. 1). The corresponding luminosity shifts from $10^{43} \mathrm{erg} / \mathrm{s}$ to $8 \times 10^{40} \mathrm{erg} / \mathrm{s}$. In particular, if one considers the case $L=2 \times 10^{42} \mathrm{erg} / \mathrm{s}$ (when following Rieger \& Mannheim (2000) BBW must be important), using Eqs. (2), (6), (12d) and (13) one can easily show that, during the course of motion, the Lorentz force always exceeds Coriolis force and the maximum Lorentz factor is limited only by ICS (see Figs. 2, 3). Moreover, from Fig. 2 it is clear that, at the beginning, the electron acceleration time scale is shorter than the cooling time scale, which means that the electron will accelerate, but, as soon as the Lorentz factor of the particle becomes of the order of $10^{5}$, the electron energy gain is counter-balanced by the losses via ICS and further acceleration is impossible.

Inverse Compton scattering of $0.1 \mathrm{keV}$ energy photons produces gamma rays with a maximum energy (Rieger \& Mannheim 2000)

$\epsilon_{\mathrm{ph}} \approx \frac{\gamma_{\max }^{2}}{10^{10}} \mathrm{TeV}$.

Considering, as an example, a luminosity power of $2 \times 10^{42} \mathrm{erg} / \mathrm{s}$, we have seen that the maximum Lorentz factor is $10^{5}$, which gives a maximum gamma ray energy of $\sim 1 \mathrm{TeV}$.

Our approach is based on the assumption that electrons corotate with the spinning AGN, which is valid only inside the Alfvén zone, because in this region the magnetic field is very strong and, as a result, the flow follows it. On the other hand, particles reach their upper energy limit in a region very close to the light cylinder, which means that our approach is valid if the following condition is satisfied: $\left(r_{\mathrm{L}}-r_{\mathrm{A}}\right) / r_{\mathrm{L}} \ll 1$ (where $r_{\mathrm{A}}$

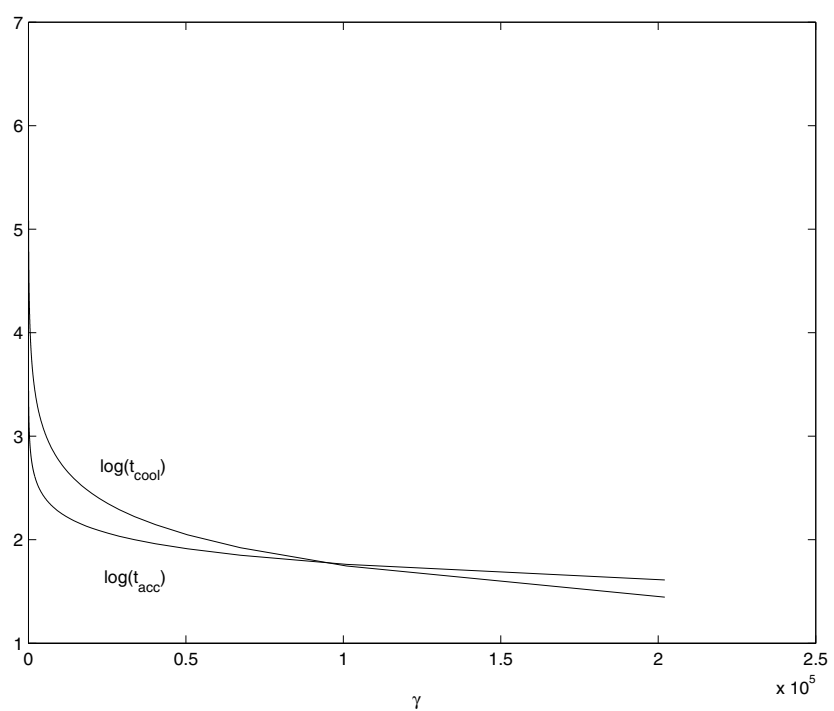

Fig. 2. Graphs of dependence of logarithms of time scales on the Lorentz factor. The set of parameters is the same as in Fig. 1, except $l=5 \times 10^{-4}$.

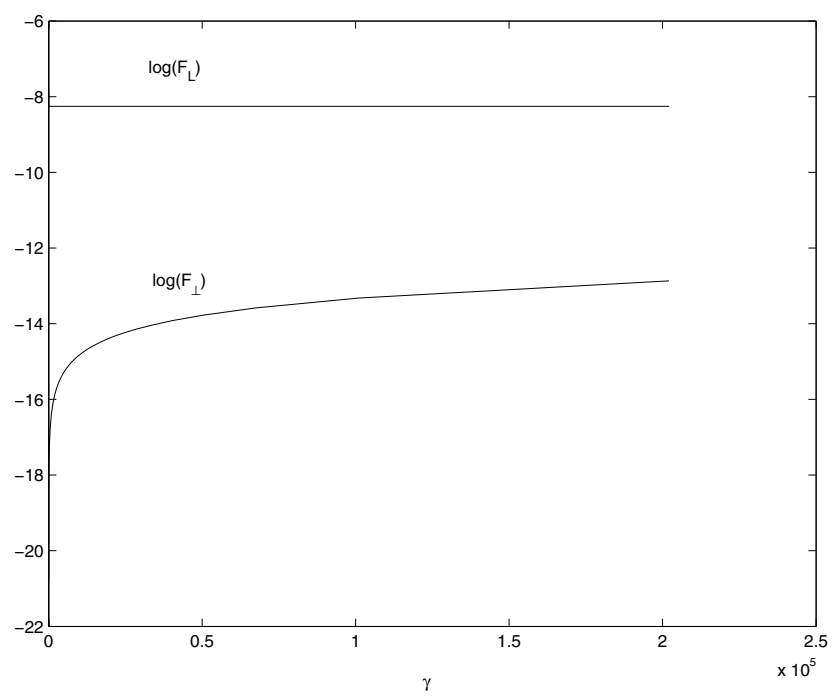

Fig. 3. Graphs of dependence of logarithms of the Lorents $F_{\mathrm{L}}$ and Coriolis $F_{\perp}$ forces on Lorentz factors. The set of parameters is the same as in Fig. 2.

is the Alfvén radius). $r_{\mathrm{A}}$ can be estimated by the expression: $B(r)^{2} /(8 \pi) \approx m_{0} n c^{2} \gamma(r)$, where $m_{0}$ and $n$ are respectively the relativistic electron's rest mass and the density. If one considers a range of $n_{J} / n_{m}=\left[10^{-4} ; 10^{4}\right]$, where $n_{J}$ is the jet bulk density and $n_{m}-$ the medium density (suppose that $n_{m} \approx 1 \mathrm{~cm}^{-3}$ ), by using Eqs. (5) and (14e) one can show that for $L=2 \times 10^{42} \mathrm{erg} / \mathrm{s}$, $\left(r_{\mathrm{L}}-r_{\mathrm{A}}\right) / r_{\mathrm{L}} \sim\left[10^{-11} ; 10^{-3}\right]$, which means that co-rotation is valid almost for the whole course of motion.

\section{The case of the inclined magnetic field lines}

In this section we examine how the situation changes when varying the inclination of magnetic field lines. For simplicity we consider the case of straight magnetic field lines.

The critical luminosity power (the luminosity power at which both mechanisms give the same maximum Lorentz factors) changes depending on the inclination angle. From the graphs of $\log \left(\gamma_{\max }^{\text {ICS }}\right)$ and $\log \left(\gamma_{\max }^{\text {BBW }}\right)$ (see Eqs. (8) and (14a)) one 


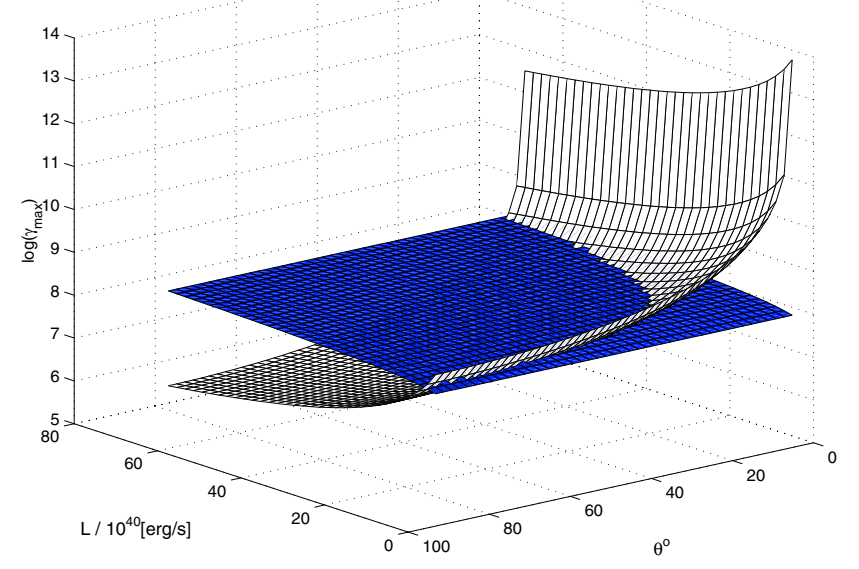

Fig. 4. Three dimensional graphs of $\log \left(\gamma_{\max }^{\mathrm{ICS}}(\theta, l)\right)$ (bright surface) and $\log \left(\gamma_{\max }^{\mathrm{BBW}}(\theta, l)\right)$ (dark surface). The set of parameters is the same as for Fig. 1 except $L$ and $\theta$ which vary respectively in ranges: $\left[5 \times 10^{40}, 7 \times\right.$ $\left.10^{41}\right] \mathrm{egr} / \mathrm{s}$ and $\theta \in\left[0.1^{\circ}, 90^{\circ}\right]$.

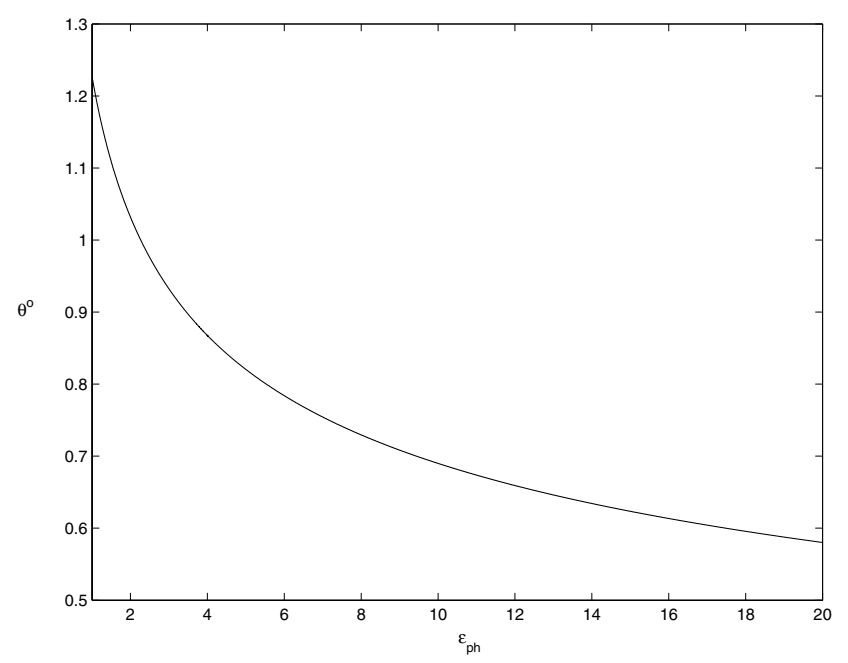

Fig. 5. Graph of dependence of $\theta\left(\epsilon_{\mathrm{ph}}\right)$ on $\epsilon_{\mathrm{ph}}$. The set of parameters is: $\omega=3 \times 10^{-5} \mathrm{~s}^{-1}$ and $L \sim 10^{44} \mathrm{erg} / \mathrm{s}$.

can see that for a luminosity less than $8 \times 10^{40} \mathrm{erg} / \mathrm{s}$ (see Fig. 1) the surface of $\log \left(\gamma_{\max }^{\text {ICS }}\right)$ is over the corresponding surface of the BBW, independent of the inclination angle (see Fig. 4). Thus, with a luminosity of $10^{41} \mathrm{erg} / \mathrm{s}$, the maximum Lorentz factor attainable by the electron is limited only by the BBW. For higher luminosity AGNs the BBW also may be important, but for relatively smaller angles (see Fig. 4).

For TeV blazars like Mrk 421 and Mrk 501 we have ultrahigh energy $\gamma$-ray emission in the range $[1-20] \mathrm{TeV}$. The luminosity power of Mrk421 and Mrk501 is estimated as $L \sim 10^{44} \mathrm{erg} / \mathrm{s}$ (Bicknell 2001). If we consider magnetic field lines inclined by the angle $\theta$, then using Eqs. (8) and (15) one may express the mentioned angle in terms of $\epsilon_{\mathrm{ph}}$ :

$\theta^{\circ} \approx \frac{4 \times 10^{3} c^{3}}{\omega L} \times\left(\frac{\epsilon_{\mathrm{ph}}}{10^{18}}\right)^{-1 / 4}$.

In Fig. 5 we show the graph of $\theta\left(\epsilon_{\mathrm{ph}}\right)$. The set of parameters is following: $\omega=3 \times 10^{-5} \mathrm{~s}^{-1}$ and $L \sim 10^{44} \mathrm{erg} / \mathrm{s}$. As it is seen from this figure, while considering magnetic field line's inclination in the range $\sim\left[0.6^{\circ}-1.2^{\circ}\right]$, one can explain the observed ultra-high energies from Mrk 421 and Mrk 501. As one can also see, by decreasing the inclination angle the corresponding energy of the photon increases. To understand the behaviour shown in Fig. 5, one has to substitute the following expressions $R_{\mathrm{L}} \approx r_{\mathrm{L}} / \sin \theta$ and $\Omega=\omega \sin \theta$ into Eqs. (2) and (6), then one gets $t_{\text {cool }} / t_{\text {acc }} \sim \Omega^{-1}$, which means that for smaller angles the ratio of time scales is higher. As a result, the particle will have more time for acceleration before energy saturation and the energy gain will be higher. That is why $\gamma_{\max }^{\mathrm{ICS}} \sim 1 / \Omega^{2}$ (see Eq. (8)), thus for smaller angles the result of acceleration is more effective than for higher inclinations, where acceleration is stronger but saturation appears very soon, hampering subsequent energy gain for particles. As a result of the angle dependence of $\gamma_{\max }^{\text {ICS }}(\theta)$, energies of produced photons become higher for smaller inclinations (see Fig. 5 and Eq. (16)).

The dependence on the angle is also very useful for studying different spinning rates. In our analysis, we considered a certain value of the angular velocity, but, on the basis of the above consideration one can predict what may happen for other values of $\omega$. As we have already seen, $\omega$ and $\theta$ are equivalent, in the sense that dynamics of particles depends on $\Omega$, but not on $\omega$ or $\theta$ independently. By applying (8) and (15) one may see $\epsilon_{\mathrm{ph}} \sim(\omega \sin \theta)^{-4}$, which means that for lower spinning rates the corresponding energies of produced photons are higher. This happens because for lower angular velocities particles have more time for acceleration and their resulting energy may be higher (see the corresponding discussion about the inclination angles).

\section{Conclusions}

In the present work we focused on the ultra-high emission from AGN, the role of the centrifugal acceleration in this process and the efficiency of different mechanisms limiting this acceleration. We re-examined and generalized the problem considered in Rieger \& Mannheim (2000), where the authors argued that together with the ICS, is also BBW an efficient limiting mechanism for electron acceleration. They argued that the BBW limits $\gamma_{\max }$ to a few thousands, which is much less than $\sim 10^{5}$ (the minimum value of the Lorentz factor needed to produce $\mathrm{TeV}$ photons).

Revisiting the same problem we showed that for AGNs considered by Rieger \& Mannheim (2000) as the acceleration limiting mechanism, only ICS is important and consequently the upper limit of the maximum Lorentz factor may be much more than several thousands. In particular, we have shown that when the magnetic field lines are straight and inclined to the axis of rotation by the angle $\theta=90^{\circ}$, for AGN with luminosity $L>8 \times 10^{40} \mathrm{erg} / \mathrm{s}$, ICS is dominant and BBW becomes significant only for AGN with lower luminosities $L<8 \times 10^{40} \mathrm{erg} / \mathrm{s}$. Therefore, the example of an AGN with a luminosity power $2 \times 10^{42} \mathrm{erg} / \mathrm{s}$, when as it was shown only ICS is important (for $\theta=90^{\circ}$ ), we calculated the approximate value of the maximum Lorentz factor of $10^{5}$ and argued that by virtue of the ICS electrons with such Lorentz factors can produce $1 \mathrm{TeV}$ energy $\gamma$-rays.

We also considered the dependence of the validity of the mentioned mechanisms on the inclination angle of the magnetic field lines with respect to the rotation axis and have shown that also in this case, for a wide range of possible inclination angles, ICS is the most important mechanism limiting the Lorentz factor. The latter value may reach up to $\sim 10^{8}$. BBW becomes dominant mostly for relatively lower luminosities with relatively higher angles, or with higher luminosities and smaller angles. The exception is the case when the luminosity is less than $8 \times 10^{40} \mathrm{erg} / \mathrm{s}$, 
when independently of the inclination angle the BBW is dominant. We can estimate by Eq. (14a) that the minimum value of $\gamma_{\max }^{\mathrm{BBW}}$ also is very high, $\sim 10^{7}$.

Letting magnetic field lines have inclination angles in the range $\sim\left[0.6^{\circ}-1.2^{\circ}\right]$, we have shown that the corresponding energies of photons due to ICS are of the order of [1-20] $\mathrm{TeV}$ which is sufficient to account for the emission of TeV blazars.

For the wide range of AGN with two considered mechanisms, ICS still remains the dominant factor in limiting the maximum Lorentz factor of accelerated particles, letting them produce very high energy photons [1-20] TeV. For low luminosity power AGN, at the other hand, BBW may be significant but it also may provide high maximum Lorentz factors of accelerated electrons.

An important restriction of our approach is that we studied only straight magnetic field lines, whereas in realistic astrophysical situations the magnetic field lines are curved. Therefore, it would be interesting to generalize our approach to how the considered problem changes, when the curvature of the magnetic field lines is taken into account. It might be especially important if we consider particle dynamics on a longer time scale, over large length-scales, when the curvature of the field lines cannot be neglected. A mathematical formalism for such a study does exist (Rogava et al. 2003) and it can be applied to the problem under consideration.

The next limitation is related to the fact that the magnetic field is not influenced by particle motion up to the region in the immediate vicinity of the light cylinder, where the magnetic field is affected by the motion of the plasma. Our model has been based on the study of the dynamics of a single particle, neglecting complex processes in real relativistic plasmas. We must generalize our approach and examine a more realistic model.

Acknowledgements. The research of Andria Rogava and Zaza Osmanov was supported in part by the Georgian National Science Foundation grant GNSF/ST06/4-096. Zaza Osmanov is grateful to Osservatorio Astronomico di Torino for the hospitality and support. Andria Rogava wishes to thank Katholieke Universiteit Leuven (Leuven, Belgium) and Abdus Salam International Centre for Theoretical Physics (Trieste, Italy) for supporting him, in part, through a Senior Postdoctoral Fellowship and Senior Associate Membership Award, respectively.

\section{References}

Aurière, M. 1982, A\&A, 109, 301

Bicknell, G., Wagner, S., \& Groves, B. 2001, AIPC, 558, 261B

Belvedere, G., Paternó, L., \& Pidatella, R. M. 1989, MNRAS, 237, 827
Blandford, R. D., \& Payne, D. G. 1982, MNRAS, 199, 883

Canizares, C. R., Grindlay, J. E., Hiltner, W. A., Liller, W., \& McClintock, J. E. 1978, ApJ, 224, 39

Catanese, M., \& Weeks, T. C. 1999, PASP, 111, 1193

Chedia, O. V., Kahniashvili, T. A., Machabeli, G. Z., \& Nanobashvili, I. S. 1996, Ap\&SS, 239, 57

Contopoulos, I., Kazanas, D., \& Fendt, C. 1999, ApJ, 511, 351

Dai, Z. G., Zhang, B., Gou, L. J., Meszaros, P., \& Waxman, E. 2002, ApJ, 580, L7

Djorgovski, S., \& King, I. R. 1984, ApJ, 277, L49

Gangadhara, R. T. 1996, A\&A, 314, 853

Gangadhara, R. T., \& Lesch, H. 1997, A\&A, 323, L45

Ghisellini, G., Haardt, F., \& Fabian, A. C. 1993, MNRAS, 263, L9

Gold, T. 1968, Nature, 218, 731

Gold, T. 1969, Nature, 221, 25

Hagiwara, K., \& Zeppenfeld, D. 1986, Nucl. Phys., 274, 1

Hauser, M. G., \& Dwek, E. 2001, ARA\&A, 39, 249

Harris, W. E., \& van den Bergh, S. 1984, AJ, 89, 1816

Hénon, M. 1961, Ann.d'Ap., 24, 369

Heiles, C., \& Troland, T. H. 2003, ApJS, 145, 329H

Horns, D. 2002, [arXiv: astro-ph/0209454]

Jimenez-Bailon, E., Santos-Lleo, M., Mas-Hesse, J. M., et al. 2003, ApJ, 591, 791

Kim, W.-T., Ostriker, E., \& Stone, J. M. 2003, ApJ, 599, 1157

King, I. R. 1966, AJ, 71, 276

King, I. R. 1975, in Dynamics of Stellar Systems, ed. A. Hayli (Dordrecht: Reidel), Proc. IAU Symp., 69, 99

King, I. R., Hedemann, E., Hodge, S. M., \& White, R. E. 1968, AJ, 73, 456

Kron, G. E., Hewitt, A. V., \& Wasserman, L. H. 1984, PASP, 96, 198

Kino, M., Takahara, F., \& Kusunose, M. 2002, ApJ, 564, 97

Kubo, H., Takahashi, T., Madejski, G., et al. 1998, ApJ, 504, 693

Lynden-Bell, D., \& Wood, R. 1968, MNRAS, 138, 495

Machabeli, G. Z., \& Rogava, A. D. 1994, Phys. Rev. A, 50, 98

Newell, E. B., \& O'Neil, E. J. 1978, ApJS, 37, 27

Okumura, K., Asahara, A., Bicknell, G. V., et al. 2002, ApJ, 579, L9-L12

Ortolani, S., Rosino, L., \& Sandage, A. 1985, AJ, 90, 473

Peterson, C. J. 1976, AJ, 81, 617

Peterson, B. M. 1997, An introduction to active galactic nuclei (Cambridge: Cambridge University Press), 34

Rieger, F. M., \& Mannheim, K. 2000, A\&A, 353, 473

Rieger, F. M., \& Duffy, P. 2004, ApJ, 617, 155

Rieger, F. M. 2005, [arXiv: astro-ph/050294]

Rogava, A. D., Dalakishvili, G., \& Osmanov, Z. N. 2003, Gen. Rel. and Grav., 35,1133

Rudnick, G., Rix, H.-W., Franx, M., et al. 2003, ApJ, 599, 847

Rybicki, G. B., \& Lightman, A. P. 1979, Radiative Processes in Astrophysics (New York: Wiley)

Spitzer, L. 1985, Dynamics of Star Clusters, ed. J. Goodman, \& P. Hut (Dordrecht: Reidel), 109

Treu, T., Ellis, R. S., Kneib, J.-P., et al. 2003, ApJ, 591, 53

Zanni, C., Massaglia, S., Bodo, G., et al. 2003, Mem. S. A. It. Suppl., 1, 155

Zanni, C., Murante, G., Bodo, G., et al. 2004,

[arXiv:astro-ph/0103200] 